CIRJE-F-857

\title{
Behavioral Aspects of Arbitrageurs in Timing Games of Bubbles and Crashes
}

\author{
Hitoshi Matsushima \\ University of Tokyo
}

August 2012

CIRJE Discussion Papers can be downloaded without charge from:

http://www.cirje.e.u-tokyo.ac.jp/research/03research02dp.html

Discussion Papers are a series of manuscripts in their draft form. They are not intended for circulation or distribution except as indicated by the author. For that reason Discussion Papers may not be reproduced or distributed without the written consent of the author. 


\title{
Behavioral Aspects of Arbitrageurs in Timing Games of Bubbles and Crashes ${ }^{1}$
}

\author{
Hitoshi Matsushima \\ Faculty of Economics, University of Tokyo \\ This Version (final): August 8, 2012 \\ First Version: March 25, 2008
}

\begin{abstract}
This paper demonstrates the theoretical foundation that underlies the willingness of rational arbitrageurs to delay and reinforce the speculative attack. The key assumptions are that there is a small probability that arbitrageurs are behavioral and never time the market of their own accord and it is uncertain whether arbitrageurs are behavioral or rational. We model a stock market as a timing game, in which arbitrageurs compete to react quickest. We show that rational arbitrageurs are willing to ride the bubble for a long period. We also characterize symmetric Nash equilibria and show the sufficient condition for uniqueness.

Keywords: Bubbles and Crashes, Timing Games, Behavioral Arbitrageurs, Reputation, Characterization, Uniqueness

JEL Classification Numbers: C720, C730, D820, G140

${ }^{1}$ This is a revised version of the manuscript entitled "Effects of Reputation in Bubbles and Crashes" (Discussion Paper CIRJE-F-560, University of Tokyo, Department of Economics, 2008). I would like to thank Professor Christian Hellwig (the editor of the Journal of Economic Theory) and Professor Marcus Brunnermeier for their helpful comments. All remaining errors are mine. This research was supported by a Grant-in-Aid for Scientific Research (Kakenhi 18330035 and 21330043) from the Japan Society for the Promotion of Science (JSPS) and the Ministry of Education, Culture, Sports, Science and Technology (MEXT).

2 Department of Economics, University of Tokyo, 7-3-1 Hongo, Bunkyo-ku, Tokyo 113-0033, Japan. Fax: +81-3-5841-5521, E-mail: hitoshi@e.u-tokyo.ac.jp.
\end{abstract}




\section{Introduction}

This paper demonstrates the theoretical foundation that underlies the willingness of rational players (arbitrageurs, portfolio managers, and investors) to delay and reinforce a speculative attack in financial markets even if they know that the mispricing is inevitably corrected in the future and they benefit by timing the market quicker than their rivals. The key assumption of this paper is incomplete information about the rationality of players, namely it is uncertain whether players are rational or not. We know that there is a small probability that a player is behavioral, in that he (she) is motivated not only by speculative interests in a sophisticated manner but also by other motives such as value storage, actual usage, and established rules of thumb. In this respect, a behavioral player is committed to never timing the market of his (her) own accord and selling up immediately after any other player sells up. This paper thus shows that behavioral type is the driving force for (i) convincing any rational player that other players are likely to delay and (ii) reinforcing the speculative attack over a long period even if all players are rational.

Specifically, this study investigates the phenomena of bubbles and crashes in a stock market by modeling a variation of the timing games (or the preemption games) with coordinated attacks presented by Abreu and Brunnermeier [1,2] and Brunnermeier and Morgan [3]. In this model, the stock market operates during the time interval $[0,1]$, and each arbitrageur selects a time to exit the market by selling up his shares. As long as no arbitrageur has yet sold up, the bubble continues to be driven by a number of positive feedback traders, who incorrectly perceive that their shares will sell for a high price and reinforce their misperception with increasing momentum. Once any arbitrageur sells up, the other arbitrageurs vie with each other to follow his example. Moreover, as soon as this pressure to sell exceeds a critical amount of shares, the positive feedback traders fail to support the stock price and the bubble crashes.

This paper assumes (i) that there is a small probability that an arbitrageur is behavioral (i.e., committed to riding the bubble at all times) and (ii) that incomplete information exists, namely whether each arbitrageur is rational or behavioral is unknown to the other arbitrageurs. On witnessing the persistence of a bubble, each 
rational arbitrageur is increasingly convinced that the other arbitrageurs are behavioral, which incentivizes him to further postpone timing the market. Based on this reasoning, each arbitrageur is convinced in the early stages of the timing game that the other arbitrageurs are likely to ride the bubble for a long period-even if they are all rational.

By restricting the growth rate of a bubble and minimizing the probability of each arbitrageur being behavioral, this paper demonstrates the existence of a unique Nash equilibrium. This symmetric equilibrium is referred to herein as the "bubble-crash equilibrium.” Where a particular point of critical time $\tilde{\tau}$ exists, (i) the bubble never crashes before the critical time $\tilde{\tau}$, whereas (ii) after the critical time $\tilde{\tau}$, the time at which the bubble crashes is randomly determined according to a constant hazard rate. This hazard rate could be very high as long as the ratio between the total amount of shares that the arbitrageurs possess and the critical amount of shares is close to unity. In this case, the bubble can persist for a long period even if all arbitrageurs are almost certain from the outset that the bubble will crash around the critical time $\tilde{\tau}$. Without these restrictions, another symmetric Nash equilibrium may exist. The present paper provides a general characterization of all symmetric Nash equilibria.

The relevance of reputation theory in finitely repeated games with incomplete information should be noted [4]. Given the assumption of incomplete information on whether players are rational or behavioral enough to have blind faith in implicit collusion, any rational player is able to mimic the collusive behavior of behavioral players. For example, Conlon [5] showed that it takes an extremely long time for any player to learn that other players are rational, which allows cooperation to persist for a long period in finitely repeated games even if all players are rational. Further, Abreu and Gul [6] applied reputation theory to a non-cooperative bargaining model in which the possibility of behavioral types in terms of strategic posture reduced the bargaining model to a war of attrition, making the bargaining outcome independent of the finer detail of the protocol.

Previous authors have also identified the conditions under which a bubble exists [1-3,7-13]. The models presented by Abreu and Brunnermeier [1,2] and Brunnermeier and Morgan [3], for instance, are particularly relevant to, but substantially different from, the model proposed in the present paper. In contrast to behavioral type, these studies introduced a rather ad hoc assumption named sequential awareness as the key 
assumption, implying that arbitrageurs become sequentially aware that a bubble has developed.

The basic notion that behavioral type incentivizes rational players to delay and reinforce the coordinated attack is so general it becomes irrelevant to the finer detail of model specifications. This point contrasts with the arguments put forward by [1-3], which depend substantially on certain model specifications, such as the exponential manner of bubble price growth. Because of this generality, we could attempt to apply it to various situations with preemptions besides bubbles and crashes left to future research. However, it would remain important to investigate bubbles and crashes further; for instance, it is implicit in the present paper to assume that arbitrageurs are prohibited from using leverage. A companion paper by Matsushima [14] modified the model by allowing arbitrageurs to use leverage under weak regulations, showing that the bubble could persist for a long period even under far weaker conditions and that it causes serious harm to society.

The remainder of this paper is organized as follows. Section 2 defines the timing games of bubbles and crashes. Section 3 characterizes the symmetric Nash equilibria by showing their necessary conditions. Section 4 examines the existence and uniqueness of the bubble-crash equilibrium. Section 5 examines other symmetric Nash equilibria. Section 6 concludes. 


\section{The Model}

Let us consider the trade in a company's shares during a time interval $[0,1]$. The fundamental value of this company is considered to be $y \geq 0$. We assume that the market interest rate is set equal to zero and no dividends are paid. There exist $n \geq 2$ arbitrageurs, each of whom decides the time to sell up his stockholding that is normalized to a single share. Let $N=\{1, \ldots, n\}$ denote the set of all arbitrageurs.

Figure 1 illustrates a typical pattern of bubbles and crashes. At the initial time 0, arbitrageurs recognize that a bubble has occurred, where the stock price is set equal to $1+y$. The bubble persists as long as at most $\tilde{n}-1$ arbitrageurs have sold up their shares, where $\tilde{n}$ is a fixed positive integer and $\tilde{n}<n$. The difference between the stock price and fundamental value grows exponentially according to a constant rate $\rho>0$; the stock price per share is considered to be $e^{\rho t}+y$ at any time $t \in[0,1]$.

\section{[Figure 1]}

Once any arbitrageur sells up his share, this pressure to sell triggers all other arbitrageurs to sell up immediately, which bursts the bubble because this collective pressure to sell exceeds the critical amount of shares $\tilde{n}$. We assume that even if no arbitrageur sells up at or before the terminal time 1, the bubble crashes immediately after the terminal time for exogenous reasons. ${ }^{3}$

Against the abovementioned background, it is implicit to assume the presence of a number of positive feedback traders who have psychological biases that lead them to engage in momentum trading. At each time $t \in[0,1]$, they incorrectly perceive that each share will sell for the price $e^{\rho t}+y$ in the future, and reinforce their misperception with increasing momentum at the rate $\rho$. The moment $\tilde{n}$ or the more the arbitrageurs sell up their shares, the more positive feedback traders fail to support the stock price,

${ }^{3}$ We assume that short selling is prohibited. Without this assumption, any single arbitrageur can burst the bubble alone. However, this case is essentially the same as the case of $\tilde{n}=1$. Hence, we could show that the bubble may survive even in this case, although this possibility is significantly limited. 
which causes it to decline to the fundamental value $y .{ }^{4}$ In this respect, our dynamic model shares aspects of coordinated attacks with the static models of currency attacks in international finance $[15,16]$. These models assume the necessity of coordination among speculators in order to break a currency peg.

This paper models the stock market as a timing game (or preemption game), where each arbitrageur as a player selects the time to sell up during the time interval [0.1]. A strategy for each arbitrageur $i \in N$ is defined as a cumulative distribution $q_{i}:[0,1] \rightarrow[0,1]$ that is nondecreasing, right continuous, and satisfies $q_{i}\left(\tau_{0}\right)=1$. Following any strategy $q_{i}$, arbitrageur $i$ plans to sell up at or before any time $a_{i} \in[0,1]$ with the probability of $q_{i}\left(a_{i}\right) \in[0,1]$. Let $Q_{i}$ denote the set of strategies for arbitrageur $i$. We consider $q_{i}=a_{i}$ to be a pure strategy, where $q_{i}(\tau)=0$ for all $\tau \in\left[0, a_{i}\right)$ and $q_{i}(\tau)=1$ for all $\tau \in\left[a_{i}, \tau_{0}\right]$.

Significantly, this paper assumes that any arbitrageur $i \in N$ is not necessarily rational; therefore, he does not necessarily follow any strategy in the set $Q_{i}$. Let us fix any arbitrary real number $\varepsilon \in[0,1]$. With regard to the probability of $\varepsilon$, arbitrageur $i$ is behavioral, in that he is committed to riding the bubble and selling up immediately after any other arbitrageur sells up. Hence, no behavioral arbitrageur can trigger bursting the bubble of his own accord. A behavioral arbitrageur can thus be regarded as being naïve enough to follow an established rule of thumb for value storage instead of pursuing speculative benefits in a sophisticated manner. ${ }^{5}$

With regard to the remaining probability of $1-\varepsilon$, arbitrageur $i$ is rational, and thus he follows a strategy in the set $Q_{i}$. Whether each arbitrageur is behavioral or rational is independently determined; therefore, no arbitrageur knows whether the other

\footnotetext{
${ }^{4}$ Abreu and Brunnermeier [1,2] assumed that whether an arbitrageur has sold up is unobservable to other arbitrageurs, whereas Brunnermeier and Morgan [3] assumed that this information is observable and thus that all arbitrageurs rush to sell up once any arbitrageur times the market. Although our paper follows the latter, we could also apply the basic concept to the former and endogenize the assumption of their rushing.

${ }^{5}$ An alternative justification is that a behavioral arbitrageur misperceives when the terminal time arrives, at which point the bubble automatically crashes. For instance, there is a dividend payment at this terminal time, triggering the crash of a bubble, but no behavioral arbitrageur knows about this dividend payment.
} 
arbitrageurs are behavioral or rational.

Suppose that each arbitrageur $i \in N$ plans to sell up at time $a_{i} \in[0,1]$. Let us arbitrarily set any nonempty subset of arbitrageurs $H \subset N$. Let us also suppose that any arbitrageur $i \in H$ is rational, while any arbitrageur $i \in N \backslash H$ is behavioral. Note that no behavioral arbitrageur $i \in N \backslash H$ sells up at his planned time $a_{i}$; instead, he is committed to waiting for any other arbitrageur to time the market. Let us denote by $\tau \in[0,1]$ the time at which any rational arbitrageur sells up first, which is defined as the earliest time at which rational arbitrageurs plan to sell up, i.e.,

$$
\tau=\min \left\{a_{i} \mid i \in H\right\} .
$$

Let $l \in\{1, \ldots,|H|\}$ denote the number of rational arbitrageurs who plan to sell up at this earliest time $\tau$, i.e.,

$$
l=\left|\left\{i \in H \mid a_{i}=\tau\right\}\right| .
$$

If $l>\tilde{n}$, then, with the probability of $\frac{\tilde{n}}{l}$, any rational arbitrageur $i \in H$ who plans to sell up at time $\tau$ can do so before the crash of the bubble and earn $e^{\rho \tau}+y$. With regard to the remaining probability of $1-\frac{\tilde{n}}{l}$, he fails to sell up before the crash and earns only the fundamental value $y$.

If $l \leq \tilde{n}$, then he can certainly sell up before the crash. In this case, $\tilde{n}-l$ further arbitrageurs can sell up before the crash; even any arbitrageur who either is behavioral or plans to sell up after time $\tau$ has the opportunity to sell up before the crash with regard to the positive probability of $\frac{\tilde{n}-l}{n-l} .^{6}$

Based on these observations, we define the expected earning of any rational arbitrageur $i \in H$ by

$$
v_{i}(H, a)=\min \left[1, \frac{K}{l}\right] e^{\rho \tau}+y \quad \text { if } a_{i}=\tau,
$$

\footnotetext{
${ }^{6}$ It is implicit to assume that behavioral arbitrageurs have the following advantage over positive feedback traders: behavioral arbitrageurs can sell up immediately after a rational arbitrageur times the market, while positive feedback traders cannot sell up until the stock price declines to the fundamental value.
} 
and

$$
v_{i}(H, a)=\max \left[\frac{K-l}{n-l}, 0\right] e^{\rho \tau}+y \quad \text { if } a_{i}>\tau .
$$

Let $Q=Q_{1} \times \cdots \times Q_{n}$. Let $q=\left(q_{1}, \ldots, q_{n}\right) \in Q$ denote a strategy profile. The payoff function $u_{i}: Q \rightarrow R$ for each arbitrageur $i \in N$ in the timing game is defined as follows; for every strategy profile $q \in Q$, we specify $u_{i}(q)$ as the expected value of $v_{i}(H, a)$ in terms of $(a, H)$, i.e.,

$$
u_{i}(q) \equiv E\left[\sum_{H \subset N: i \in H} v_{i}(H, a) \varepsilon^{n-|H|}(1-\varepsilon)^{|H|-1} \mid q\right],
$$

where $E[\cdot \mid q]$ is the expectation operator in terms of $a \equiv\left(a_{j}\right)_{j \in N} \in[0,1]^{n}$, and note that the probability that $H \backslash\{i\}$ is the set of all rational arbitrageurs except arbitrageur $i$ equals $\varepsilon^{n-|H|}(1-\varepsilon)^{|H|-1}$. A strategy profile $q \in Q$ is said to be symmetric if

$$
q_{i}=q_{1} \text { for all } i \in N \text {. }
$$

A strategy profile $q \in Q$ is said to be a Nash equilibrium in the timing game if

$$
u_{i}(q) \geq u_{i}\left(q_{i}^{\prime}, q_{-i}\right) \text { for all } i \in N \text { and all } q_{i}^{\prime} \in Q_{i} .
$$

Let us introduce several notations as follows. For each strategy profile $q \in Q$, let us denote the probability that the bubble has crashed at or before any time $t \in[0,1]$ by

$$
D(t ; q) \equiv 1-\prod_{i \in N}\left[\varepsilon+(1-\varepsilon)\left\{1-q_{i}(t)\right\}\right] .
$$

Note that $D(1 ; q) \equiv 1-\varepsilon^{n}<1$ whenever $\varepsilon>0$, which implies that the bubble does not necessarily crash during the time interval $[0,1]$ because there is a positive probability of $\varepsilon^{n}>0$ that all arbitrageurs are behavioral. Let us denote the hazard rate at which the bubble crashes at any time $t \in[0,1]$ by

$$
\theta(t ; q) \equiv \frac{\frac{\partial D(t ; q)}{\partial t}}{1-D(t ; q)} .
$$

Moreover, for each arbitrageur $i \in N$ and each strategy profile $q \in Q$, let us denote the probability that the bubble has crashed at or before any time $t$, provided arbitrageur $i$ never bursts the bubble of his own accord, by 


$$
D_{i}\left(t ; q_{-i}\right)=1-\prod_{j \in N \backslash\{i\}}\left[\varepsilon+(1-\varepsilon)\left\{1-q_{j}(t)\right\}\right] .
$$

The notation of $D_{i}\left(t ; q_{-i}\right)$ will be helpful to investigate the Nash equilibrium property, because, as shown in the next section, the strategy choices $q_{-i}$ by the other arbitrageurs influence arbitrageur i's payoff $u_{i}(q)$ only through $D_{i}\left(t ; q_{-i}\right)$. 


\section{Necessary Condition for Symmetric Nash Equilibrium}

We arbitrarily set a time $\hat{\tau} \in[0,1]$, where we assume that

$$
\max \left[0,1+\frac{(n-\tilde{n}) \ln \varepsilon}{\rho}\right] \leq \hat{\tau} \leq 1 .
$$

Let us specify a real number $k(\hat{\tau}) \in[0,1]$ by

$$
k(\hat{\tau}) \equiv \frac{1-\varepsilon \exp \left[\frac{\rho(1-\hat{\tau})}{n-\tilde{n}}\right]}{1-\varepsilon},
$$

where inequality (1) guarantees $k(\hat{\tau}) \in[0,1]$. Let us specify a symmetric strategy profile, denoted by $q^{\hat{\tau}}=\left(q_{i}^{\hat{\tau}}\right)_{i \in N} \in Q$, as follows: for every $i \in N$,

$$
q_{i}^{\hat{\imath}}\left(a_{i}\right)=k(\hat{\tau}) \text { for all } a_{i} \in[0, \hat{\tau}] \text {, }
$$

and

$$
q_{i}^{\hat{\tau}}\left(a_{i}\right)=\frac{1-\varepsilon \exp \left[\frac{\rho\left(1-a_{i}\right)}{n-\tilde{n}}\right]}{1-\varepsilon} \text { for all } a_{i} \in[\hat{\tau}, 1],
$$

where specification (2) implies $k(\hat{\tau})=q_{i}^{\hat{\tau}}(\hat{\tau})$, i.e., $q_{i}^{\hat{\tau}}$ is continuous. According to $q^{\hat{\tau}}$, any rational arbitrageur $i \in N$ plans to sell up at the initial time 0 with a probability of $k(\hat{\tau})$. With regard to the remaining probability of $1-k(\hat{\tau})$, he plans to ride the bubble up to time $\hat{\tau}$.

The following proposition shows that any symmetric Nash equilibrium $q \in Q$ belongs to the class of symmetric strategy profiles specified in the above manner.

Proposition 1: If any strategy profile $q \in Q$ is a symmetric Nash equilibrium in the timing game, then there exists $\hat{\tau} \in[0,1]$ such that $q=q^{\hat{\tau}}$.

Proof: We set any symmetric Nash equilibrium $q \in Q$ arbitrarily, where we assume that $q \neq q^{1}$, i.e., $q_{1}(0)<1$.

We show that $q_{1}(\tau)$ is continuous in $[0,1]$. Suppose that $q_{1}(\tau)$ is not continuous in $[0,1]$, i.e., there exists $\tau^{\prime}>0$ such that $\lim _{\tau \uparrow \tau^{\prime}} q_{1}(\tau)<q_{1}\left(\tau^{\prime}\right)$. Since 
$\min \left[1, \frac{\tilde{n}}{l+1}\right]-\max \left[0, \frac{\tilde{n}-l}{n-l}\right]>0$ for all $l \in\{0, \ldots, n-1\}$, it follows from the symmetry of $q$ that by selecting any time that is slightly earlier than time $\tau^{\prime}$, any arbitrageur can drastically increase the probability of his winning the timing game. This implies that no arbitrageur selects time $\tau^{\prime}$, which is a contradiction.

Let us specify

$$
\hat{\tau}=\max \left\{\tau \in(0,1]: q_{1}(\tau)=q_{1}(0)\right\} .
$$

We show that $q_{1}(\tau)$ is increasing in $[\hat{\tau}, 1]$. Suppose that $q_{1}(\tau)$ is not increasing in $[\hat{\tau}, 1]$. From the continuity of $q_{1}$ and specification of $\hat{\tau}$, we can select $\tau^{\prime}, \tau^{\prime \prime} \in[\hat{\tau}, 1]$ such that $\tau^{\prime}<\tau^{\prime \prime}, q_{1}\left(\tau^{\prime}\right)=q_{1}\left(\tau^{\prime \prime}\right)$, and the time choice $\tau^{\prime}$ is a best response. Since no arbitrageur selects any time $\tau$ in $\left(\tau^{\prime}, \tau^{\prime \prime}\right)$, it follows from the continuity of $q$ that by selecting time $\tau^{\prime \prime}$ instead of $\tau^{\prime}$, any arbitrageur can increase the winner's gain from $e^{\rho \tau^{\prime}}+y$ to $e^{\rho \tau^{\prime \prime}}+y$ without decreasing his winning probability. This is a contradiction.

Note that any time choice $\tau \in[\hat{\tau}, 1]$ is a best response, because $q_{1}(\tau)$ is increasing in $[\hat{\tau}, 1]$. This implies the following first-order conditions for all $\tau \in[\hat{\tau}, 1]$ :

$$
\frac{\partial u_{1}\left(\tau, q_{-1}\right)}{\partial \tau}=\rho e^{\rho \tau}\left\{1-D_{1}\left(\tau ; q_{-1}\right)\right\}-e^{\rho \tau} \frac{\tilde{n}-1}{n-1} \frac{d D_{1}\left(\tau ; q_{-1}\right)}{d \tau}=0,
$$

i.e.,

$$
D_{1}\left(\tau ; q_{-1}\right)=1-C e^{\frac{-\rho \tau}{1-\lambda}},
$$

where $C$ is a positive real number. Since $q$ is symmetric and continuous, it follows that

$$
D_{1}\left(1 ; q_{-1}\right)=1-C e^{\frac{-\rho}{1-\lambda}}=1-\varepsilon^{n-1},
$$

and

$$
D_{1}\left(\hat{\tau} ; q_{-1}\right)=1-C e^{\frac{-\rho \tau^{1}}{1-\lambda}}=D_{1}\left(0 ; q_{-1}\right) .
$$

From (3), it follows that

$$
C=\varepsilon^{n-1} e^{\frac{\rho}{1-\lambda}}
$$

and therefore, 
(5)

$$
D_{1}\left(\tau ; q_{-1}\right)=1-\varepsilon^{n-1} e^{\frac{\rho(1-\tau)}{1-\lambda}} \text { for all } \tau \in[\hat{\tau}, 1] .
$$

Since $q$ is symmetric, it follows from equalities (4) and (5) that

$$
q_{i}^{\hat{\tau}}\left(a_{i}\right)=\frac{1-\varepsilon \exp \left[\frac{\rho(1-\hat{\tau})}{n-\tilde{n}}\right]}{1-\varepsilon} \text { for all } a_{i} \in[0, \hat{\tau}),
$$

and

$$
q_{i}^{\hat{\imath}}\left(a_{i}\right)=\frac{1-\varepsilon \exp \left[\frac{\rho\left(1-a_{i}\right)}{n-\tilde{n}}\right]}{1-\varepsilon} \text { for all } a_{i} \in[\hat{\tau}, 1],
$$

which implies that $q=q^{\hat{\hat{\imath}}}$.

Q.E.D.

Proposition 1 implies that in any symmetric Nash equilibrium $q$, the shape of the function $D(t ; q)$ corresponds to Figure 2, where $q=q^{\hat{\imath}}$, and for every $t \in[0,1]$,

$$
D(t ; q)=1-\varepsilon^{n} e^{\frac{n}{n-\tilde{n}} \rho(1-\hat{\tau})} \text { and } \theta(t ; q)=0 \quad \text { if } 0 \leq t<\hat{\tau},
$$

and

$$
D(t ; q)=1-\varepsilon^{n} e^{\frac{n}{n-\tilde{n}} \rho(1-t)} \quad \text { and } \theta(t ; q)=\frac{n \rho}{n-\tilde{n}} \quad \text { if } \quad \hat{\tau}<t \leq 1 .
$$

After the critical time $\hat{\tau}$, the time at which the bubble crashes is randomly determined according to a constant hazard rate $\frac{n \rho}{n-\tilde{n}}$.

\section{[Figure 2]}

According to the standard method of the preemption game analysis, the proof of Proposition 1 showed that $D(t ; q)$ must be continuous in $t$ and that there must exist $\hat{\tau}$ such that $D(t ; q)$ is constant in $[0, \hat{\tau}]$, whereas $D(t ; q)$ is increasing in $[\hat{\tau}, 1]$ and that during the interval $[\hat{\tau}, 1]$ the first-order condition must hold. These three properties imply that any symmetric Nash equilibrium corresponds to that in Figure 2. 


\section{Bubble-Crash Equilibrium}

This section assumes that the growth rate of a bubble $\rho>0$ and the probability of each arbitrageur being behavioral $\varepsilon \in(0,1)$ satisfies that

$$
\varepsilon^{n-\tilde{n}} e^{\rho} \geq 1 \text {, i.e., } 0 \leq 1+\frac{(n-\tilde{n}) \ln \varepsilon}{\rho}<1,
$$

which implies that $\rho$ and $\varepsilon$ are not very small. Let us specify a particular time $\tilde{\tau} \in[0,1)$ by

$$
\tilde{\tau} \equiv 1+\frac{(n-\tilde{n}) \ln \varepsilon}{\rho},
$$

where inequality (6) guarantees $\tilde{\tau} \in[0,1)$. Note that

$$
k(\tilde{\tau})=0 .
$$

Let us define the bubble-crash strategy profile by $\tilde{q}=\left(\tilde{q}_{i}\right)_{i \in N} \equiv\left(q_{i}^{\tilde{\tau}}\right)_{i \in N} \in Q$ : for every $i \in N$,

$$
\tilde{q}_{i}\left(a_{i}\right)=k(\tilde{\tau})=0 \text { for all } a_{i} \in[0, \tilde{\tau}] \text {, }
$$

and

$$
\tilde{q}_{i}\left(a_{i}\right)=\frac{1-\varepsilon \exp \left[\frac{\rho\left(1-a_{i}\right)}{n-\tilde{n}}\right]}{1-\varepsilon} \text { for all } a_{i} \in[\tilde{\tau}, 1] .
$$

According to the bubble-crash strategy profile $\tilde{q}$, the bubble never crashes before the critical time $\tilde{\tau}$.

Theorem 2: With inequality (6), the bubble-crash strategy profile $\tilde{q}$ is a Nash equilibrium, and

$$
u_{i}(\tilde{q})=\varepsilon^{n-\tilde{n}} e^{\rho}+y .
$$

Proof: Since the bubble never crashes before time $\tilde{\tau}$, it follows from the continuity of $\tilde{q}$ that

$$
u_{i}\left(a_{i}, \tilde{q}_{-i}\right)=e^{\rho a_{i}}+y \text { for all } a_{i} \in[0, \tilde{\tau}) .
$$

Since $\tilde{q}$ is continuous, it follows that 


$$
u_{i}\left(\tilde{\tau}, \tilde{q}_{-i}\right)=e^{\rho \tilde{\tau}}+y>u_{i}\left(a_{i}, \tilde{q}_{-i}\right) \text { for all } a_{i} \in[0, \hat{\tau}) .
$$

Since any time choice is indifferent in $[\hat{\tau}, 1]$, i.e.,

$$
u_{i}\left(\hat{\tau}, q_{-i}^{\hat{\tau}}\right)=u_{i}\left(a_{i}, q_{-i}^{\hat{\tau}}\right) \text { for all } i \in N \text { and all } a_{i} \in[\hat{\tau}, 1],
$$

it follows that

$$
u_{i}\left(\tilde{\tau}, \tilde{q}_{-i}\right)=u_{i}\left(a_{i}, \tilde{q}_{-i}\right) \text { for all } a_{i} \in[\tilde{\tau}, 1] .
$$

Hence, we have proven that $\tilde{q}$ is a Nash equilibrium. From (7),

$$
u_{i}\left(\tilde{\tau}, \tilde{q}_{-i}\right)=e^{\rho \tilde{\tau}}+y=\varepsilon^{n-\tilde{n}} e^{\rho}+y,
$$

which along with $u_{i}(\tilde{q})=u_{i}\left(\tilde{\tau}, \tilde{q}_{-i}\right)$ implies equality (9).

\section{Q.E.D.}

Note that the probability $D(t ; \tilde{q})$ that the bubble crashes at or before any time $t \in[0,1]$ is given by

$$
D(t ; \tilde{q})=0 \quad \text { if } 0 \leq t<\tilde{\tau},
$$

and

$$
D(t ; \tilde{q})=1-\varepsilon^{n} e^{\frac{n}{n-\tilde{n}} \rho(1-t)} \quad \text { if } \tilde{\tau}<t \leq 1 .
$$

See Figure 6, which illustrates $D(t ; \tilde{q})$.

\section{[Figure 3]}

From the specification of $\tilde{q}$, it follows

$$
\frac{\frac{\partial \tilde{q}_{i}(t)}{\partial t}}{1-\tilde{q}_{i}(t)}=\frac{\frac{\rho}{n-\tilde{n}} \exp \left[\frac{\rho(1-t)}{n-\tilde{n}}\right]}{\exp \left[\frac{\rho(1-t)}{n-\tilde{n}}\right]-1} .
$$

Note $\lim _{t \downarrow \hat{\tau}} \frac{\frac{\partial \tilde{q}_{i}(t)}{\partial t}}{1-\tilde{q}_{i}(t)}=0$, which implies that around the critical time $\hat{\tau}$, any rational arbitrageur almost certainly postpones timing the market. Note also $\lim _{t \uparrow_{1}} \frac{\frac{\partial \tilde{q}_{i}(t)}{\partial t}}{1-\tilde{q}_{i}(t)}=+\infty$, 
which implies that as the terminal time 1 draws near, any rational arbitrageur is in a great hurry to time the market. Hence, the reason why even rational arbitrageurs have an incentive to ride the bubble is as follows, which is similar to reputation theory in finitely repeated games:

(i) As terminal time 1 draws near, any arbitrageur is almost convinced that the other arbitrageurs are behavioral.

(ii) At any time around the critical time $\hat{\tau}$, any arbitrageur is convinced through his rational reasoning that the other arbitrageurs are likely to ride the bubble even if they are rational.

Note that the hazard rate after the critical time $\hat{\tau}$, i.e., $\frac{n \rho}{n-\tilde{n}}$, diverges to infinity as the ratio between the total amount of shares that the arbitrageurs possess and the critical amount of shares, i.e., $\frac{\tilde{n}}{n}$, approaches unity. Note also that the critical time $\tilde{\tau}$ depends not on the ratio $\frac{\tilde{n}}{n}$, but on $(n-\tilde{n}) \ln \varepsilon$. This implies that the bubble can persist for a long period even if all arbitrageurs are almost certain that it will crash around a particular fixed time $\tilde{\tau}$.

The following theorem shows a sufficient condition under which the bubble-crash strategy profile $\tilde{q}$ is the unique Nash equilibrium, even if we take into account all asymmetric Nash equilibria.

Theorem 3: The bubble-crash strategy profile $\tilde{q}$ is the unique Nash equilibrium in the timing game if

$$
\varepsilon^{n-1} e^{\rho}>1
$$

Proof: See the On-Line Appendices.

A brief illustration of the proof follows. Inequality (10) implies that the time choice 0 is a dominated strategy, which along with Proposition 1 implies that $\tilde{q}$ is the unique symmetric Nash equilibrium. We can prove the continuity and increasing 
property of any asymmetric Nash equilibrium $q$ in the same manner as the proof of Proposition 1. We can show that any Nash equilibrium $q$ must be symmetric as follows. The first-order condition implies

$$
\frac{\frac{\partial D_{i}(t ; q)}{\partial t}}{1-D_{i}(t ; q)}=\rho \frac{n-1}{\tilde{n}-1} \text { for all } i \in N \text { and all } t \in\left[\tau^{1}, 1\right] \text {. }
$$

Inequality (10) implies that the time choice 0 is dominated, i.e.,

$$
q_{i}\left(\tau^{1}\right)=0 \text { for all } i \in N,
$$

which along with (11) implies that $q_{i}=q_{1}$ for all $i \in N$, i.e., $q$ must be symmetric. ${ }^{7}$

7 Without inequality (10), we may not be able to show this symmetry; however, we may also not be able to exclude the possibility that any asymmetric Nash equilibrium $q$ exists, such that $q_{i}(0)>0$ and $q_{i}(0) \neq q_{j}(0)$ for some $i \in N$ and some $j \in N \backslash\{i\}$. 


\section{Other Symmetric Nash Equilibria}

We denote by $q^{*} \equiv q^{1}$ the quick-crash strategy profile: for every $i \in N$,

$$
q_{i}^{*}\left(a_{i}\right)=q_{i}^{1}\left(a_{i}\right)=k(1)=1 \text { for all } i \in N \text { and all } a_{i} \in[0,1] \text {. }
$$

According to $q^{*}$, any rational arbitrageur plans to sell up at the initial time 0 ; the bubble never persists as long as there is a rational arbitrageur.

Proposition 4: The quick-crash strategy profile $q^{*}$ is a Nash equilibrium if and only if

$$
\begin{aligned}
& \sum_{\substack{H \subset N \\
i \notin H, H \neq \phi}} \frac{(n-1) !}{|H| !(n-1-|H|) !}(1-\varepsilon)^{|H|} \varepsilon^{n-1-|H|}\left\{\min \left[1, \frac{\tilde{n}}{|H|+1}\right]-\max \left[0, \frac{\tilde{n}-|H|}{n-|H|}\right]\right\} \\
\geq & \varepsilon^{n-1}\left(e^{\rho}-1\right) .
\end{aligned}
$$

Proof: See the On-Line Appendices.

Note that $q^{*}$ is a Nash equilibrium if $\varepsilon=0$, i.e., it is certain that all arbitrageurs are rational. In this case, the left-hand side of (13) equals zero, while its right-hand side equals $\min \left[1, \frac{\tilde{n}}{n}\right]>0$, which automatically implies inequality (13). The assumption of $\varepsilon=0$ also implies the uniqueness of the Nash equilibrium; any rational arbitrageur dislikes losing the opportunity to become the single winner of the timing game. This presses him to react slightly earlier than the others. This aspect of tail-chasing competition eliminates all equilibria other than $q^{*}$.

This logic, however, cannot be applied to the case of $\varepsilon>0$. Even if any arbitrageur plans to sell up at the terminal time 1, he still has the opportunity of becoming the single winner with regard to the positive probability $\varepsilon^{n}$; the assumption of $\varepsilon>0$ may prevent their tail-chasing competition.

Let us investigate symmetric strategy profiles $q^{\hat{\tau}}$ besides the bubble-crash strategy profile $\tilde{q}$ and the quick-crash strategy profile $q^{*}$. These can be termed hybrid strategy profiles. When a hybrid strategy profile is played, the advantage of riding the 
bubble is severely limited because the time choice 0 is a best response, i.e., $u_{i}\left(q^{\hat{\tau}}\right)=u_{i}\left(0, q_{-i}^{\hat{\tau}}\right)<1+y$. Note that $\hat{\tau}>\tilde{\tau}$, which implies that once the bubble takes off, it tends to grow further than that induced by the bubble-crash equilibrium. The following proposition shows the necessary and sufficient condition under which a hybrid strategy profile $q^{\hat{\tau}}$ is a Nash equilibrium.

Proposition 5: The hybrid strategy profile $q^{\hat{\imath}}$ is a Nash equilibrium if and only if

$$
\begin{aligned}
& \{1-(1-\varepsilon) k(\hat{\tau})\}^{n-1}\left\{e^{\rho}\left(\frac{\varepsilon}{1-(1-\varepsilon) k(\hat{\tau})}\right)^{n-\tilde{n}}-1\right\} \\
& =\sum_{\substack{H \in N: \\
i \notin H, H \neq \phi}}\left[\frac{(n-1) !}{|H| !(n-1-|H|) !}\{(1-\varepsilon) k(\hat{\tau})\}^{|H|}\{1-(1-\varepsilon) k(\hat{\tau})\}^{n-1-|H|}\right. \\
& \left.\cdot\left\{\min \left[1, \frac{\tilde{n}}{|H|+1}\right]-\max \left[0, \frac{\tilde{n}-|H|}{n-|H|}\right]\right\}\right] .
\end{aligned}
$$

Proof: See the On-Line Appendices.

The following theorem shows that if neither the quick-crash equilibrium $q^{*}$ nor the bubble-crash equilibrium $\tilde{q}$ exists, then there exists $\hat{\tau} \in(\tilde{\tau}, 1)$ such that the corresponding hybrid strategy profile $q^{\hat{\tau}}$ is a Nash equilibrium.

Theorem 6: If neither inequality (6) nor inequality (13) holds, then there exists $\hat{\tau} \in(\tilde{\tau}, 1)$ such that $q^{\hat{\imath}}$ is a Nash equilibrium.

Proof: For every $h \in[0,1]$, let us define

$$
\begin{aligned}
& B(h) \equiv\{1-(1-\varepsilon) h\}^{n-1}\left\{e^{\rho}\left(\frac{\varepsilon}{1-(1-\varepsilon) h}\right)^{n-\tilde{n}}-1\right\} \\
& -\sum_{\substack{H \subset N: \\
i \notin H, H \neq \phi}}\left[\frac{(n-1) !}{|H| !(n-1-|H|) !}\{(1-\varepsilon) h\}^{|H|}\{1-(1-\varepsilon) h\}^{n-1-H \mid}\right.
\end{aligned}
$$




$$
\left.\cdot\left\{\min \left[1, \frac{\tilde{n}}{|H|+1}\right]-\max \left[0, \frac{\tilde{n}-|H|}{n-|H|}\right]\right\}\right] .
$$

Note that $B(h)$ is continuous. Since inequality (6) does not hold, it follows that $B(0)<0$. Since inequality (13) does not hold, it follows that $B(1)>0$. These imply that there exists $h \in(0,1)$ such that $B(h)=0$. This implies equality (14).

Q.E.D.

Note that when $\tilde{n}$ is sufficiently large, there is no possibility that neither inequality (6) nor inequality (13) holds. However, even in this case, there is a possibility that the hybrid equilibrium exists; the following proposition shows the multiplicity in that if the quick-crash and bubble-crash equilibria coexist, then there also exists another hybrid Nash equilibrium.

Proposition 7: If inequalities (6) and (13) hold with strict inequality, then there exists $\hat{\tau} \in(\tilde{\tau}, 1)$ such that $q^{\hat{\tau}}$ is a Nash equilibrium.

Proof: Note that the function $B(h)$ defined in the proof of Theorem 6 is continuous. Since inequality (6) holds with strict inequality, it follows that $B(0)>0$. Since inequality (13) holds with strict inequality, it follows that $B(1)<0$. These imply that there exists $h \in(0,1)$ such that $B(h)=0$. This implies equality (14).

Q.E.D. 


\section{Conclusion}

This paper modeled a stock market as a timing game with incomplete information, in which arbitrageurs competed to react quickest during the bubble persisting. We assumed that there was a small probability that they were behavioral. As a theoretical foundation underlying bubbles and crashes, we showed that rational arbitrageurs were willing to ride the bubble for a long period as their Nash equilibrium behavior. 


\section{References}

[1] D. Abreu, M. Brunnermeier, Synchronization risk and delayed arbitrage, J. Finan. Econ. 66 (2002), 341-360.

[2] D. Abreu, M. Brunnermeier, Bubbles and crashes, Econometrica 71 (2003), 173-204.

[3] M. Brunnermeier, J. Morgan, Clock games: Theory and experiments, mimeo, 2006.

[4] D. Kreps, P. Milgrom, J. Roberts, R. Wilson, Rational cooperation in the finitely repeated prisoners’ dilemma, J. Econ. Theory 27 (1982), 245-252, 486-502.

[5] J. Conlon, Hope springs eternal: Learning and the stability of cooperation in short horizon repeated games, J. Econ. Theory 112 (2003), 35-65.

[6] D. Abreu, F. Gul, Bargaining and reputation, Econometrica 68 (2000), 85-117.

[7] J. Harrison, D. Kreps, Speculative investor behavior in a stock market with heterogeneous expectations, Quart. J. Econ. 92 (1978), 323-336.

[8] O. Blanchard, M. Watson, Bubbles, rational expectations, and financial markets, in: P. Wachtel (Ed.), Crisis in Economic and Financial Structure, Lexington Books, Lexington, MA, 1982, pp. 295-315.

[9] J. Tirole, On the possibility of speculation under rational expectations, Econometrica 50 (1982), 1163-1181.

[10] J. Tirole, Asset bubbles and overlapping generations, Econometrica, 53 (1985), 1499-1528.

[11] F. Allen, G. Gordon, Churning bubbles, Rev. Econ. Stud. 60 (1993), 813-836.

[12] F. Allen, S. Morris, A. Postlewaite, Finite bubbles with short sale constraints and asymmetric information,” J. Econ. Theory 61 (1993), 206-229.

[13] J. Scheinkman, W. Xiong, Overconfidence and speculative bubbles, J. Polit. Economy 111 (2003), 1183-1219.

[14] H. Matsushima, H. Financing harmful bubbles, Discussion Paper CIRJE-F-756, University of Tokyo, Department of Economics, 2010.

[15] S. Morris, H. Shin, Unique equilibrium in a model of self-fulfilling currency attacks, Amer. Econ. Rev. 88 (1998), 587-596.

[16] M. Obstfeld, Models of currency crises with self-fulfilling features,” Europ. Econ. 
Rev. 40 (1996), 1037-1047. 
Figure 1

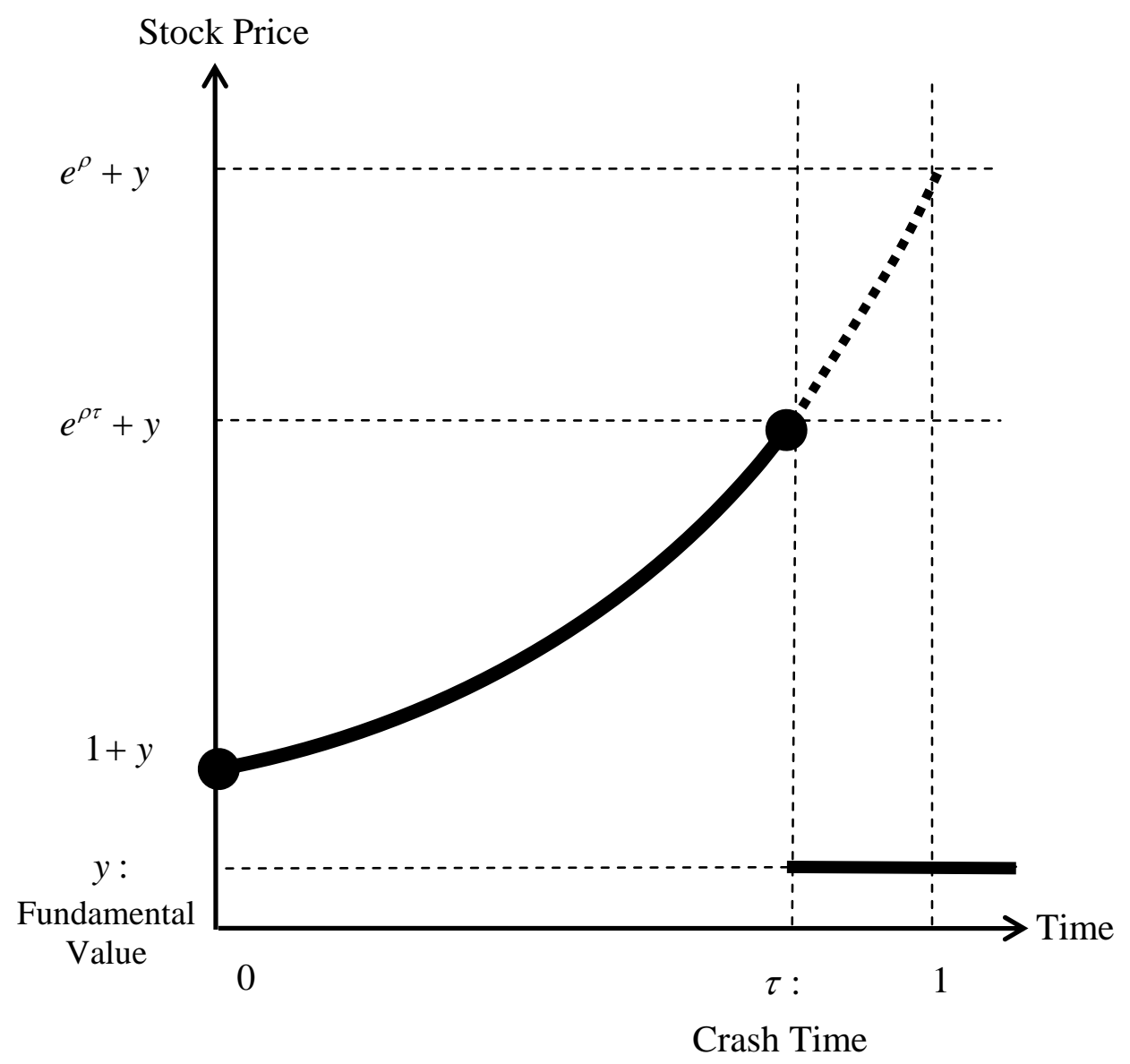


Figure 2

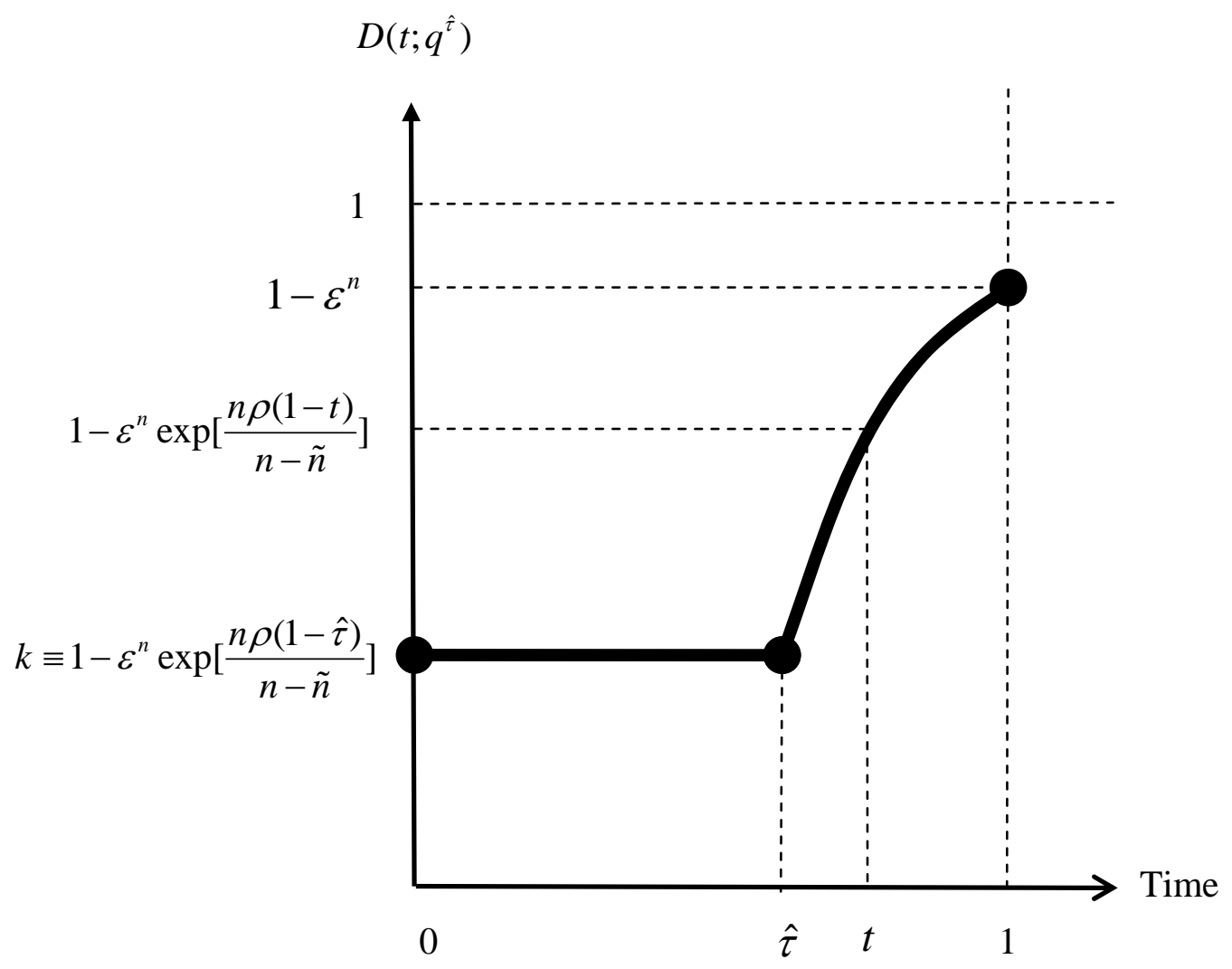


Figure 3

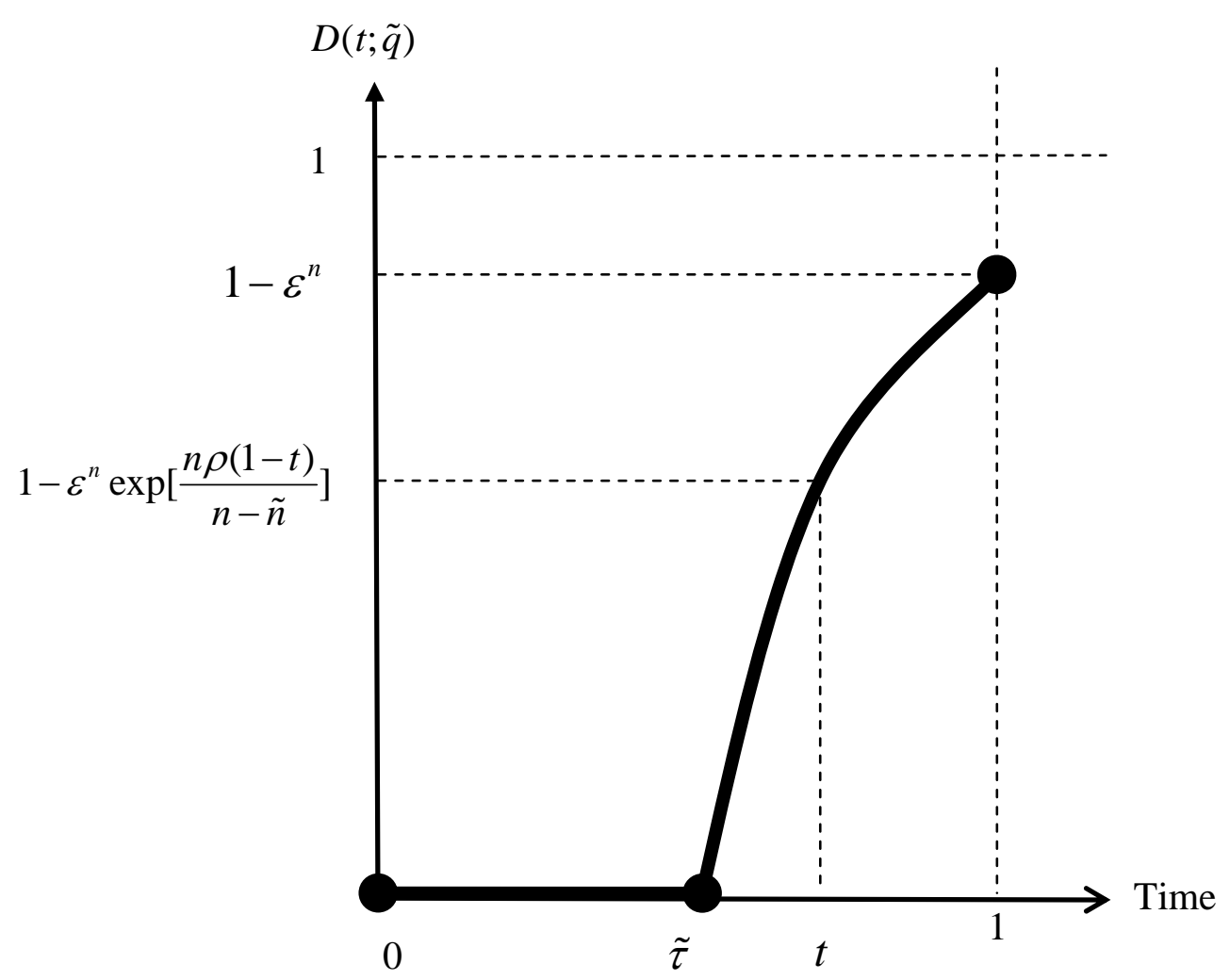




\title{
On-Line Appendices to
}

\section{Behavioral Aspects of Arbitrageurs in Timing Games of Bubbles and Crashes}

\author{
Hitoshi Matsushima \\ Faculty of Economics, University of Tokyo \\ 7-3-1 Bunkyo-ku, Tokyo 113-0033, Japan. hitoshi@e.u-tokyo.ac.jp
}

This Version (final): August 8, 2012

First Version: March 25, 2008

Proof of Theorem 3: We now show that $\tilde{q}$ is the unique symmetric Nash equilibrium. Note that

$$
u_{1}\left(0, q_{-1}^{\prime}\right) \leq 1+y \text { and } u_{1}\left(1, q_{-1}^{\prime}\right) \geq \varepsilon^{n-1} e^{\rho}+y \text { for all } q_{-1}^{\prime} \in Q_{-1} .
$$

This along with inequality (10) implies that the time choice of 0 is dominated by the time choice of 1, i.e.,

$$
u_{1}\left(1, q_{-1}^{\prime}\right)>u_{1}\left(0, q_{-1}^{\prime}\right) \text { for all } q_{-1}^{\prime} \in Q_{-1} .
$$

Hence, any symmetric Nash equilibrium $q \in Q$ must satisfy $q_{1}(0)=0$, which along with Proposition 1 implies $q=\tilde{q}$. Since inequality (10) implies inequality (6), we have proven that $\tilde{q}$ is the unique symmetric Nash equilibrium.

We next show that $\tilde{q}$ is the unique Nash equilibrium, even if all asymmetric Nash equilibria are taken into account. We set any Nash equilibrium $q \in Q$ arbitrarily.

First, we show that $q_{i}(\tau)$ must be continuous in [0,1] for all $i \in N$. Suppose that $q_{i}(\tau)$ is not continuous in $[0,1]$. Then, there exists $\tau^{\prime}>0$ such that $\lim _{\tau \uparrow} q_{i}(\tau)<q_{i}\left(\tau^{\prime}\right)$ 
for some $i \in N$. Since $\min \left[1, \frac{\tilde{n}}{l+1}\right]-\max \left[0, \frac{\tilde{n}-l}{n-l}\right]>0$ for all $l \in\{0, \ldots, n-1\}$, it follows that any other arbitrageur can drastically increase his winning probability by selecting any time that is slightly earlier than time $\tau^{\prime}$. Hence, no other arbitrageur ever selects any time that is either the same as, or slightly later than, time $\tau^{\prime}$. This implies that arbitrageur $i$ can increase the winner's gain by postponing timing the market further without decreasing his winning probability. This is a contradiction.

Second, we show that $D(\tau ; q)$ must be increasing in $\left[\tau^{1}, 1\right]$, where we denote

$$
\tau^{1}=\max \left\{\tau \in(0,1]: q_{i}(\tau)=q_{i}(0) \text { for all } i \in N\right\} .
$$

Suppose that $D(\tau ; q)$ is not increasing in $\left[\tau^{1}, 1\right]$. Hence, from the continuity of $q$, we can select $\tau^{\prime}, \tau^{\prime \prime} \in\left(\tau^{1}, 1\right]$ such that $\tau^{\prime}<\tau^{\prime \prime}, D\left(\tau^{\prime} ; q\right)=D\left(\tau^{\prime \prime} ; q\right)$, and the time choice $\tau^{\prime}$ is a best response for some arbitrageur. Since no arbitrageur selects any time $\tau$ in $\left(\tau^{\prime}, \tau^{\prime \prime}\right)$, it follows from the continuity of $q$ that by selecting time $\tau^{\prime \prime}$ instead of $\tau^{\prime}$, any arbitrageur can increase the winner's gain from $e^{\rho \tau^{\prime}}+y$ to $e^{\rho \tau^{\prime \prime}}+y$ without decreasing his winning probability. This is a contradiction.

Third, we show that $q$ must be symmetric. Suppose that $q$ is asymmetric. Since inequality (10) implies that the time choice 0 is a dominated strategy, it follows $\tau^{1}>0$, and

$$
q_{i}(\tau)=0 \text { for all } i \in N \text { and all } \tau \in\left[0, \tau^{1}\right]
$$

Since $q$ is continuous and $D(\tau ; q)$ is increasing in $\left[\tau^{1}, 1\right]$, from the supposition $\tau^{1}>0$ and equality (A-1), it follows that there exist $\tau^{\prime}>0, \tau^{\prime \prime}>\tau^{\prime}$, and $i \in N$ such that

$$
\begin{aligned}
q_{1}(t) & =q_{j}(t) \text { for all } j \in N \text { and all } t \in\left[0, \tau^{\prime}\right], \\
& \frac{\frac{\partial D_{i}(\tau ; q)}{\partial t}}{1-D_{i}(\tau ; q)}>\min _{h \neq i} \frac{\frac{\partial D_{h}(\tau ; q)}{\partial t}}{1-D_{h}(\tau ; q)} \text { for all } t \in\left(\tau^{\prime}, \tau^{\prime \prime}\right),
\end{aligned}
$$

and

$$
\frac{\frac{\partial D_{i}\left(\tau^{\prime \prime} ; q\right)}{\partial t}}{1-D_{i}\left(\tau^{\prime \prime} ; q\right)}=\min _{h \neq i} \frac{\frac{\partial D_{h}\left(\tau^{\prime \prime} ; q\right)}{\partial t}}{1-D_{h}\left(\tau^{\prime \prime} ; q\right)}>0
$$


where the last inequality was derived from the increasing property of $D(\tau ; q)$ in $\left[\tau^{1}, 1\right]$. Since $D(\tau ; q)$ is increasing in $\left[\tau^{1}, 1\right]$, any time choice $t$ in $\left(\tau^{\prime}, \tau^{\prime \prime}\right)$ must be a best response for any arbitrageur $j \in N$ satisfying that

$$
\frac{\frac{\partial D_{j}(t ; q)}{\partial t}}{1-D_{j}(t ; q)}=\min _{h \neq i} \frac{\frac{\partial D_{h}(t ; q)}{\partial t}}{1-D_{h}(t ; q)} .
$$

Since this equality implies $\frac{\partial q_{j}(t)}{\partial t}>0$, it follows from the continuity of $q$ that the first-order condition holds for arbitrageur $j$; for every $t \in\left(\tau^{\prime}, \tau^{\prime \prime}\right)$,

$$
\frac{\partial u_{j}\left(\tau, q_{-j}\right)}{\partial \tau}=\rho e^{\rho \tau}\left\{1-D_{j}\left(\tau ; q_{-j}\right)\right\}-e^{\rho \tau} \frac{\tilde{n}-1}{n-1} \frac{d D_{j}\left(\tau ; q_{-j}\right)}{d \tau}=0,
$$

i.e.,

$$
\rho \frac{n-1}{\tilde{n}-1}=\min _{h \neq i} \frac{\frac{\partial D_{h}(t ; q)}{\partial t}}{1-D_{h}(t ; q)} .
$$

Hence, from (A-2),

$$
\rho \frac{n-1}{\tilde{n}-1}<\frac{\frac{\partial D_{i}(t ; q)}{\partial t}}{1-D_{i}(t ; q)},
$$

which implies that the first-order condition does not hold for arbitrageur $i$ for every $t \in\left(\tau^{\prime}, \tau^{\prime \prime}\right)$, where

$$
\frac{\partial u_{i}\left(\tau, q_{-i}\right)}{\partial \tau}=\rho e^{\rho \tau}\left\{1-D_{i}\left(\tau ; q_{-i}\right)\right\}-e^{\rho \tau} \frac{\tilde{n}-1}{n-1} \frac{d D_{i}\left(\tau ; q_{-i}\right)}{d \tau}<0 .
$$

This inequality implies that arbitrageur $i$ prefers time $\tau^{\prime}$ rather than any time in $\left(\tau^{\prime}, \tau^{\prime \prime}+\varepsilon\right)$, and therefore,

$$
\frac{\partial D_{i}(\tau ; q)}{\partial t}=0 \text { for all } \tau \in\left(\tau^{\prime}, \tau^{\prime \prime}+\varepsilon\right)
$$

where $\varepsilon$ was positive but close to zero. This is a contradiction, because the inequality of (A-3) implied $\frac{\partial D_{i}\left(\tau^{\prime \prime} ; q\right)}{\partial t}>0$. Hence, we have proven that any Nash equilibrium $q$ must be symmetric.

Q.E.D. 
Proof of Proposition 4: For every $i \in N$,

$$
u_{i}\left(q^{*}\right)=\sum_{\substack{H \subset N \\ i \notin H, H \neq \phi}} \frac{(n-1) !}{|H| !(n-1-|H|) !}(1-\varepsilon)^{|H|} \varepsilon^{n-1-|H|} \min \left[1, \frac{\tilde{n}}{|H|+1}\right]+\varepsilon^{n-1}+y,
$$

and for every $a_{i} \in(0,1]$,

$$
\begin{aligned}
& u_{i}\left(a_{i}, q_{-i}^{*}\right)=\sum_{\substack{H \subset N \\
i \notin H, H \neq \phi}} \frac{(n-1) !}{|H| !(n-1-|H|) !}(1-\varepsilon)^{|H|} \varepsilon^{n-1-|H|} \max \left[0, \frac{\tilde{n}-|H|}{n-|H|}\right] \\
& +\varepsilon^{n-1} e^{\rho a_{i}}+y .
\end{aligned}
$$

Hence, for every $a_{i} \in(0,1]$,

$$
\begin{aligned}
& u_{i}\left(q^{*}\right)-u_{i}\left(a_{i}, q_{-i}^{*}\right) \\
& =\sum_{\substack{H \subset N \\
i \notin H, H \neq \phi}} \frac{(n-1) !}{|H| !(n-1-|H|) !}(1-\varepsilon)^{|H|} \varepsilon^{n-1-|H|}\left\{\min \left[1, \frac{\tilde{n}}{|H|+1}\right]-\max \left[0, \frac{\tilde{n}-|H|}{n-|H|}\right\}\right. \\
& +\varepsilon^{n-1}\left(1-e^{\rho a_{i}}\right) .
\end{aligned}
$$

From $e^{\rho} \geq e^{\rho a_{i}}$ for all $a_{i} \in(0,1]$, and from the above observations, it follows that inequality (13) is necessary and sufficient for $q^{*}$ to be a Nash equilibrium.

\section{Q.E.D.}

Proof of Proposition 5: From the specification of $q^{\hat{\tau}}$, it follows that $u_{i}\left(0, q_{-i}^{\hat{\tau}}\right)$ equals

$$
\begin{aligned}
& \sum_{\substack{H \in N: \\
i \notin H, H \neq \phi}} \frac{(n-1) !}{|H| !(n-1-|H|) !}\{(1-\varepsilon) k(\hat{\tau})\}^{|H|}\{1-(1-\varepsilon) k(\hat{\tau})\}^{n-1-|H|} \min \left[1, \frac{\tilde{n}}{|H|+1}\right] \\
& +\{1-(1-\varepsilon) k(\hat{\tau})\}^{n-1}+y,
\end{aligned}
$$

and for every $a_{i} \in(0,1], u_{i}\left(a_{i}, q_{-i}^{\hat{\tau}}\right)$ equals

$$
\begin{aligned}
& \sum_{\substack{H \subset N: \\
i \notin H, H \neq \phi}} \frac{(n-1) !}{|H| !(n-1-|H|) !}\{(1-\varepsilon) k(\hat{\tau})\}^{|H|}\{1-(1-\varepsilon) k(\hat{\tau})\}^{n-1-|H|} \max \left[0, \frac{\tilde{n}-|H|}{n-|H|}\right] \\
& +\{1-(1-\varepsilon) k(\hat{\tau})\}^{n-1} e^{\rho a_{i}}+y \quad \text { if } a_{i} \in(0, \hat{\tau}),
\end{aligned}
$$

and 
(A-6)

$$
\begin{aligned}
& \sum_{\substack{H \in N: \\
i \notin H, H \neq \phi}} \frac{(n-1) !}{|H| !(n-1-|H|) !}\{(1-\varepsilon) k(\hat{\tau})\}^{|H|}\{1-(1-\varepsilon) k(\hat{\tau})\}^{n-1-H \mid} \max \left[0, \frac{\tilde{n}-|H|}{n-|H|}\right] \\
& +e^{\rho a_{i}}\left\{1-D_{i}\left(a_{i} ; q_{-i}^{k}\right)\right\}+\frac{\tilde{n}-1}{n-1} \int_{t=0}^{a_{i}} e^{\rho t} d D_{i}\left(t ; q_{-i}^{k}\right)+y \quad \text { if } a_{i} \in[\hat{\tau}, 1] .
\end{aligned}
$$

Moreover, from the specification of $q^{\hat{\imath}}$,

$$
D_{i}\left(t ; q_{-i}^{\hat{\tau}}\right)=1-\{\varepsilon+(1-\varepsilon)(1-k(\hat{\tau}))\}^{n-1} \text { for all } t \in[0, \hat{\tau}),
$$

and

$$
D_{i}\left(t ; q_{-i}^{\hat{\tau}}\right)=1-\varepsilon^{n-1} e^{\frac{n-1}{n-\tilde{n}} \rho(1-t)} \text { for all } t \in[\hat{\tau}, 1] .
$$

Since $\tilde{q}$ is continuous, it follows that for every $a_{i} \in(0, \hat{\tau})$,

$$
u_{i}\left(\hat{\tau}, q_{-i}^{\hat{\tau}}\right)-u_{i}\left(a_{i}, q_{-i}^{\hat{\tau}}\right)=\{1-(1-\varepsilon) k(\hat{\tau})\}^{n-1}\left(e^{\rho \hat{\imath}}-e^{\rho a_{i}}\right)>0 .
$$

From (A-4) and (A-5), for every $i \in N$ and every $a_{i} \in(0, \hat{\tau})$,

$$
\begin{aligned}
& u_{i}\left(0, q_{-i}^{\hat{\tau}}\right)-u_{i}\left(a_{i}, q_{-i}^{\hat{\tau}}\right) \\
& =\sum_{\substack{H \in N: \\
i \notin H, H \neq \phi}} \frac{(n-1) !}{|H| !(n-1-|H|) !}\{(1-\varepsilon) k(\hat{\tau})\}^{|H|}\{1-(1-\varepsilon) k(\hat{\tau})\}^{n-1-H \mid} \\
& \left\{\min \left[1, \frac{\tilde{n}}{|H|+1}\right]-\max \left[0, \frac{\tilde{n}-|H|}{n-|H|}\right]\right\}+\{1-(1-\varepsilon) k(\hat{\tau})\}^{n-1}\left(1-e^{\rho a_{i}}\right)>0 .
\end{aligned}
$$

From equalities (2) and (14), it follows that for every $i \in N$,

$$
\begin{aligned}
& u_{i}\left(0, q_{-i}^{\hat{\tau}}\right)-u_{i}\left(\hat{\tau}, q_{-i}^{\hat{\tau}}\right) \\
& =\sum_{\substack{H \subset N \\
i \notin H, H \neq \phi}} \frac{(n-1) !}{|H| !(n-1-|H|) !}\{(1-\varepsilon) k(\hat{\tau})\}^{|H|}\{1-(1-\varepsilon) k(\hat{\tau})\}^{n-1-|H|} \\
& \left\{\min \left[1, \frac{\tilde{n}}{|H|+1}\right]-\max \left[0, \frac{\tilde{n}-|H|}{n-|H|}\right]\right\}+\{1-(1-\varepsilon) k(\hat{\tau})\}^{n-1}\left(1-e^{\rho \hat{\tau}}\right)=0,
\end{aligned}
$$

where the last equality was derived from (2) and (14). From (A-6), the following first-order condition holds for every $a_{i} \in[\hat{\tau}, 1]$;

$$
\begin{aligned}
& \frac{\partial}{\partial a_{i}} u_{i}\left(a_{i}, q_{-i}^{\hat{\tau}}\right)=\frac{\partial}{\partial a_{i}}\left[e^{\rho a_{i}}\left\{1-D_{i}\left(a_{i} ; q_{-i}^{\hat{\tau}}\right)\right\}+\frac{\tilde{n}-1}{n-1} \int_{t=0}^{a_{i}} e^{\rho t} d D_{i}\left(t ; q_{-i}^{\hat{\tau}}\right)\right] \\
& =\rho e^{\rho a_{i}}\left\{1-D_{i}\left(a_{i} ; q_{-i}^{\hat{\imath}}\right)\right\}-\frac{n-\tilde{n}}{n-1} e^{\rho a_{i}} \frac{\partial D_{i}\left(a_{i} ; q_{-i}^{\hat{\tau}}\right)}{\partial a_{i}}
\end{aligned}
$$




$$
=\rho e^{\rho a_{i}} \varepsilon^{n-1} e^{\frac{n-1}{n-\tilde{n}} \rho(1-t)}-\frac{n-\tilde{n}}{n-1} e^{\rho a_{i}} \frac{\partial D_{i}\left(a_{i} ; q_{-i}^{\hat{\tau}}\right)}{\partial a_{i}}=0,
$$

where, from (A-7), we have derived

$$
\frac{\partial D_{i}\left(a_{i} ; q_{-i}^{\hat{\tau}}\right)}{\partial a_{i}}=\frac{n-1}{n-\tilde{n}} \rho \varepsilon^{n-1} e^{\frac{n-1}{n-\tilde{n}} \rho(1-t)},
$$

which implies the last equality of (A-8). Hence,

$$
u_{i}\left(\hat{\tau}, q_{-i}^{\hat{\tau}}\right)=u_{i}\left(a_{i}, q_{-i}^{\hat{\tau}}\right) \text { for all } a_{i} \in[\hat{\tau}, 1],
$$

and therefore, we have proven that

$$
u_{i}\left(q^{\hat{\tau}}\right) \geq u_{i}\left(q_{i}, q_{-i}^{\hat{\tau}}\right) \text { for all } q_{i} \in Q_{i} \text { and all } i \in N .
$$

Q.E.D. 